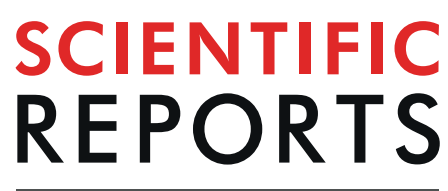

natureresearch

\title{
Unravelling the mechanism of pressure induced polyamorphic transition in an inorganic molecular glass
}

\author{
Bora Kalkan ${ }^{1,2^{*}}$, Gokce Okay ${ }^{3}$, Bruce G. Aitken ${ }^{4}$, Simon M. Clark ${ }^{5,6}$ \& Sabyasachi Sen ${ }^{7}$ \\ The atomic structure of a germanium doped phosphorous selenide glass of composition $\mathrm{Ge}_{2.8} \mathrm{P}_{57.7} \mathrm{Se}_{39.5}$ \\ is determined as a function of pressure from ambient to $24 \mathrm{GPa}$ using Monte-Carlo simulations \\ constrained by high energy $x$-ray scattering data. The ambient pressure structure consists primarily \\ of $\mathrm{P}_{4} \mathrm{Se}_{3}$ molecules and planar edge shared phosphorus rings, reminiscent of those found in red \\ phosphorous as well as a small fraction of locally clustered corner-sharing $\mathrm{GeSe}_{4}$ tetrahedra. This \\ low-density amorphous phase transforms into a high-density amorphous phase at $\sim 6.3 \mathrm{GPa}$. The high- \\ pressure phase is characterized by an extended network structure. The polyamorphic transformation \\ between these two phases involves opening of the $\mathrm{P}_{3}$ ring at the base of the $\mathrm{P}_{4} \mathrm{Se}_{3}$ molecules and \\ subsequent reaction with red phosphorus type moieties to produce a cross linked structure. The \\ compression mechanism of the low-density phase involves increased molecular packing, whereas \\ that of the high pressure phase involves an increase in the nearest-neighbor coordination number \\ while the bond angle distributions broaden and shift to smaller angles. The entropy and volume \\ changes associated with this polyamorphic transformation are positive and negative, respectively, \\ and consequently the corresponding Clapeyron slope for this transition would be negative. This result \\ has far reaching implications in our current understanding of the thermodynamics of polyamorphic \\ transitions in glasses and glass-forming liquids.
}

The existence and the nature of polyamorphic phase transitions in the glassy or liquid state between structurally and thermodynamically distinct phases of the same composition remain controversial issues in the fields of condensed matter physics and chemistry ${ }^{1}$. The most extensively studied case of polyamorphism in the literature pertains to the transitions between the low- and high- density phases of $\mathrm{H}_{2} \mathrm{O}$ in the supercooled liquid state ${ }^{2-4}$. The low-density phase is characterized by an open, hydrogen-bonded tetrahedral "molecular" structure with low entropy, which transforms under pressure into a high-density network structure with high entropy ${ }^{4}$. This inverse relation between volume and entropy implies a negative slope in the P-T space for this polyamorphic transition. However, the structural complexity of water has made the experimental determination of the atomistic mechanism of this structural transformation rather difficult. On the other hand, structurally simple van der Waals -bonded molecular liquids may prove to be more tractable systems for studying the atomistic mechanisms of molecular-to-network polyamorphic transformations.

A handful of inorganic crystals are known to be molecular and consist of nearly spherical cage-like molecules held together by the van der Waals force; examples include $\mathrm{As}_{4} \mathrm{~S}_{3}, \mathrm{P}_{4} \mathrm{~S}_{3}, \mathrm{P}_{4} \mathrm{Se}_{3}$ and $\mathrm{C}_{60}$. It has been demonstrated in the literature that, amongst these compositions, $\mathrm{As}_{4} \mathrm{~S}_{3}$ and $\mathrm{P}_{4} \mathrm{Se}_{3}$ can be glass-forming if the liquid is stabilized against crystallization by the addition of a small concentration of $\mathrm{Ge}$ and, in the case of $\mathrm{P}_{4} \mathrm{Se}_{3}$, by excess phosphorus. These glasses, consisting predominantly of isolated cage molecules, constitute ideal systems for mechanistic studies of molecular-to-network polyamorphic transformation. Recently, we have reported the observation of

\footnotetext{
${ }^{1}$ Earth and Planetary Sciences Department, University of California, Santa Cruz, CA, 95064, USA. ${ }^{2}$ Advanced Light Source, Lawrence Berkeley National Laboratory, Berkeley, CA, 94720, USA. ${ }^{3}$ Department of Physics Engineering, Hacettepe University, Ankara, 06800, Beytepe, Turkey. ${ }^{4}$ Glass Research Division, Corning Inc., Corning, New York, 14831, USA. ${ }^{5}$ Department of Earth and Environmental Sciences, Macquarie University, North Ryde, NSW, Australia. ${ }^{6}$ School of Engineering, Macquarie University, North Ryde, NSW, 2109, Australia. ${ }^{7}$ Department of Materials Science and Engineering, University of California-Davis, Davis, California, 95616, USA. *email: bkalkan@lbl.gov.tr
} 

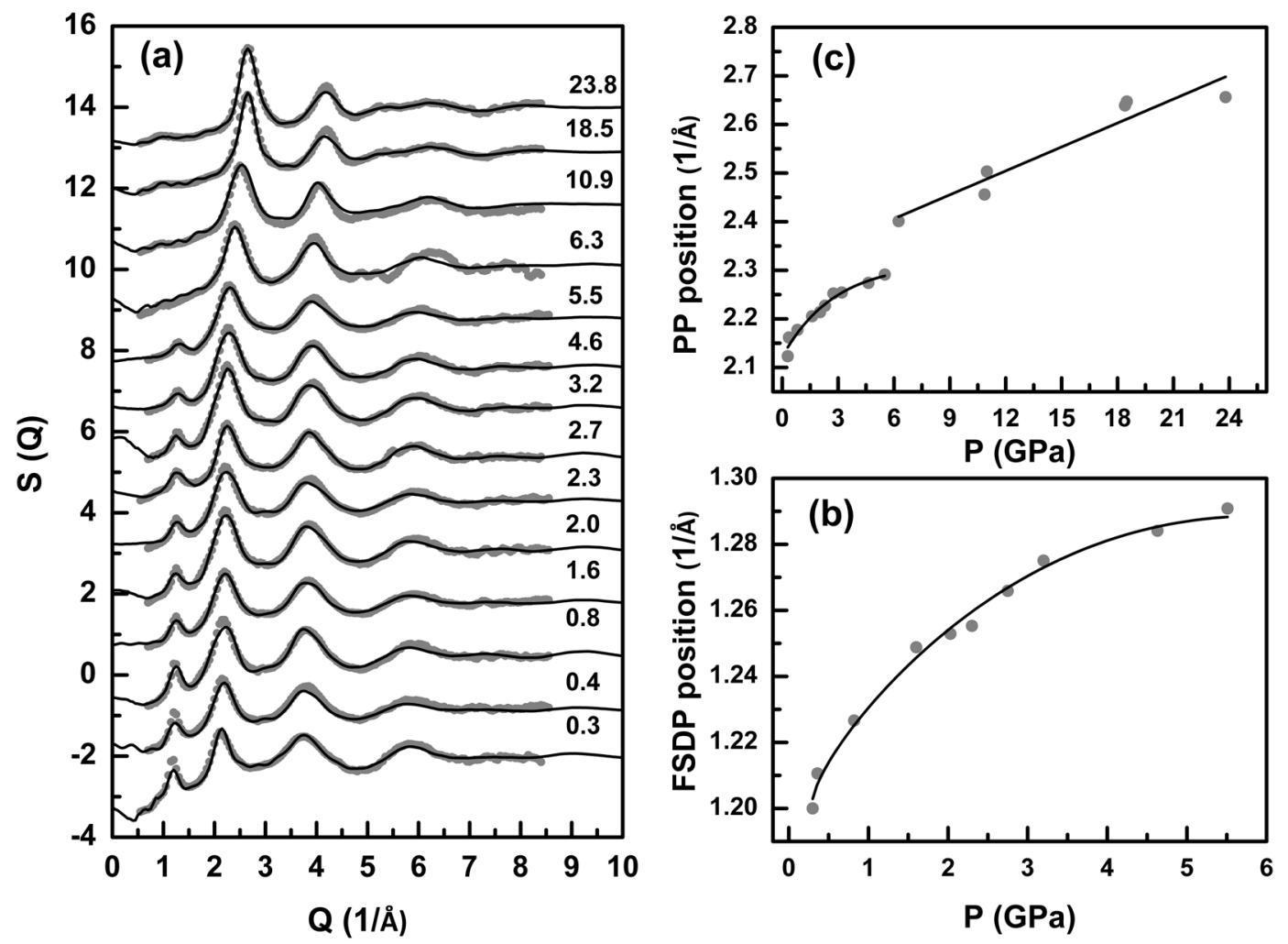

Figure 1. High pressure $x$-ray scattering data. (a) Evolution of $S(Q)$ during compression. Experimental (thick gray dots) and EPSR simulated (black lines) total structure factors at different pressures. Data are vertically offset to enhance clarity and corresponding pressures in GPa are given alongside each pattern. (b) Pressure dependence of the position of the FSDP. (c) Pressure dependence of the position of the PP in the S(Q). Solid lines through the data points in (b) and (c) are guides to the eye.

a pressure-induced polyamorphic transition in a chalcogenide glass of composition $\mathrm{Ge}_{2.5} \mathrm{As}_{51.25} \mathrm{~S}_{46.25}$ at ambient temperature ${ }^{5-7}$. In situ high-pressure $\mathrm{x}$-ray scattering, absorption and Raman spectroscopic measurements indicated a hysteretically reversible transformation between a low-density molecular structure and a high-density network structure near $\sim 2 \mathrm{GPa}$. However, the atomistic mechanism of this structural transformation had remained speculative.

Similar predominantly molecular glasses in the P-Se system near the $\mathrm{P}_{5} \mathrm{Se}_{3}$ composition with and without $\mathrm{Ge}$-doping have been reported in the literature ${ }^{8-10}$. Structural studies of these glasses based on Raman and ${ }^{31} \mathrm{P}$ nuclear magnetic resonance (NMR) spectroscopy have indicated the presence of $\mathrm{P}_{4} \mathrm{Se}_{3}$ cage molecules and amorphous red-phosphorus type moieties ${ }^{8,10}$. In this study, we have used a combination of state-of-the art high energy $\mathrm{X}$-ray diffraction and 3D Monte Carlo structural modeling to investigate the structure of such a glass of composition $\mathrm{Ge}_{2.8} \mathrm{P}_{57.7} \mathrm{Se}_{39.5}$ (denoted henceforth as GPS, for the sake of brevity) and its pressure-dependent evolution in situ. The primary goal of this comprehensive study is to obtain a direct understanding of the short and intermediate -range structural aspects and the atomistic mechanism of the pressure-induced molecular-to-network transformation in this glass.

\section{Results}

Evolution of the structure factor $\mathrm{S}(\mathrm{Q})$ at pressures ranging between $0.3 \mathrm{GPa}$ and $24 \mathrm{GPa}$. The evolution of the $\mathrm{S}(\mathrm{Q})$ of GPS glass with pressure upon compression up to $23.8 \mathrm{GPa}$ is shown in Fig. 1a. The $\mathrm{S}(\mathrm{Q})$ is characterized by an intense first sharp diffraction peak (FSDP), located at $\mathrm{Q} \sim 1.18 \AA^{-1}$ signifying intermediate range order at a length scale of $\sim 5.3 \AA$, resulting from strong intermolecular correlations in these glasses ${ }^{11,12}$. The chemical and topological ordering in the structure is characterized by the principal peak (PP) and the third peak, respectively ${ }^{13}$. One of the most dramatic changes in the total experimental $S(Q)$ with increasing pressure is a rapid decrease in the intensity of the FSDP. In fact, the FSDP practically disappears completely at pressures above 6.3 GPa (Fig. 1b). During decompression from $23.8 \mathrm{GPa}$, a hysteretic behavior is observed as the FSDP reappears only when the sample is fully decompressed (Supplementary Fig. S1), although the original intensity of the uncompressed sample is never recovered. On the other hand, the FSDP position rapidly increases to higher Q with pressure in the low-pressure regime below $\sim 1.5 \mathrm{GPa}$ and the slope abruptly changes at higher pressures with a slower rate of increase above $\sim 1.5-2.0 \mathrm{GPa}$ (Fig. 1b). In contrast with the behavior of the FSDP, the intensity of the $\mathrm{PP}$ increases with increasing pressure (Fig. 1c) and its position shifts to higher Q values. The PP shifts from $\sim 2.10 \AA^{-1}$ at ambient pressure to $\sim 2.65 \AA^{-1}$ at $23.8 \mathrm{GPa}$ and its intensity increases by a factor of $\sim 2.4$. A closer look at the pressure dependence of the PP position shows that the PP position rapidly increases to higher $\mathrm{Q}$ with 

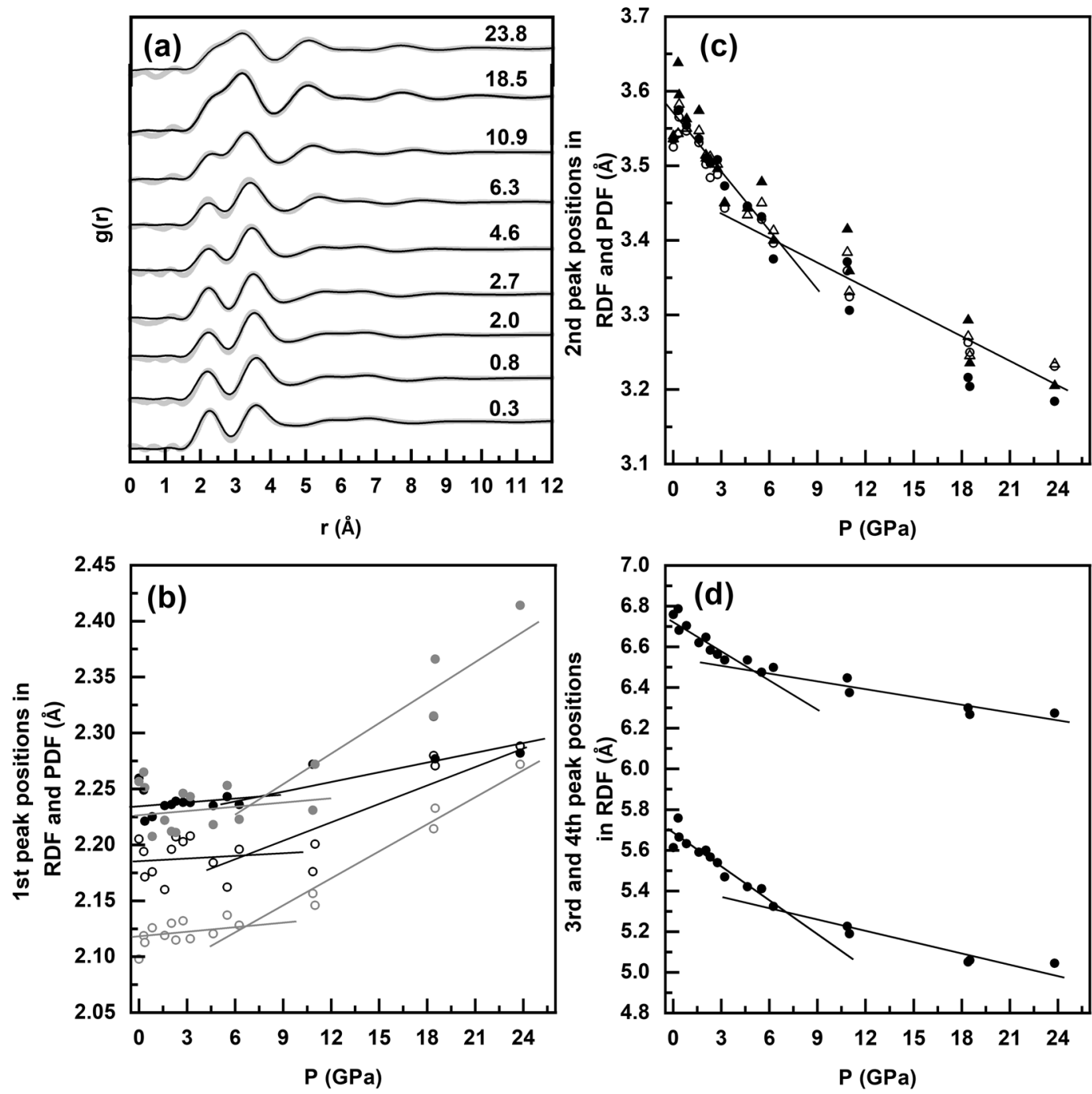

Figure 2. Pressure dependence of RDF. (a) Pressure dependence of experimental (gray trace) and simulated (black lines) g(r) during compression of the GPS glass. Data are vertically offset for clarity and corresponding pressures in GPa are given alongside each pattern. (b) Pressure dependence of the first peak positions in total $\mathrm{g}$ (r) (black dots), and in the P-P (black open circles), P-Se (gray dots), and Ge-Se (gray open circles) PDFs. (c) The second peak positions in total g(r) (black dots) and in the P-P (black solid triangles), P-Se (black open triangles), and Se-Se (black open circles) PDFs. (d) The third and fourth peak positions in total g(r).

pressure in the low-pressure regime below $\sim 2.5$ to $3.0 \mathrm{GPa}$, followed by a slower rate of increase at higher pressures up to $\sim 6.3 \mathrm{GPa}$. Further increase in pressure results in an abrupt jump in PP position to $2.4 \AA^{-1}$ at $\sim 6.3 \mathrm{GPa}$ followed by a smooth and monotonic increase in PP position between $\sim 6.3$ and $23.8 \mathrm{GPa}$.

Structure analysis in real space under pressure. The experimental and simulated radial distribution functions (RDFs) obtained during compression of the GPS glass are compared in Fig. 2a. Constrained EPSR g(r) data enable a rigorous and comprehensive interpretation of short- and intermediate- range structural evolution with pressure. It is clear from Fig. 2a that the qualitative appearance of the RDFs in the high-pressure regime $(>6.3 \mathrm{GPa})$ are distinctly different from those in the low-pressure regime $(<6.3 \mathrm{GPa})$. The pressure dependence of all of the peaks in the RDFs and the corresponding $\mathrm{Ge}-\mathrm{Se}, \mathrm{Se}-\mathrm{Se}, \mathrm{P}-\mathrm{Se}$ and $\mathrm{P}-\mathrm{P}$ pair distribution functions (PDFs) (Supplementary Fig. S2) determined from the EPSR simulations (Fig. 2b-d) indicates that the intermolecular correlation peaks rapidly shift to shorter distances up to $\sim 6.3 \mathrm{GPa}$ (Fig. $2 \mathrm{~d}$ ). This result suggests that the densification mechanism in this pressure range is dominated by increased molecular packing ${ }^{14}$. The rate of this pressure-induced shift in the peak positions abruptly changes with a slower rate of decrease at pressures above 6.3 GPa. In contrast, the average nearest-neighbor distance in $\mathrm{g}(\mathrm{r})$, and the P-Se, P-P and Ge-Se nearest-neighbor peaks in the EPSR PDFs exhibit a slight elongation over the entire pressure range (Fig. 2b). The g(r) curves of GPS glass obtained upon decompression are depicted in Supplementary Fig. S3a. A closer look at the g(r) patterns indicate that the disordered phase obtained after the compression-decompression cycle is somewhat denser than the starting phase (Supplementary Fig. S3b) with slightly shorter intermolecular correlation distances. 

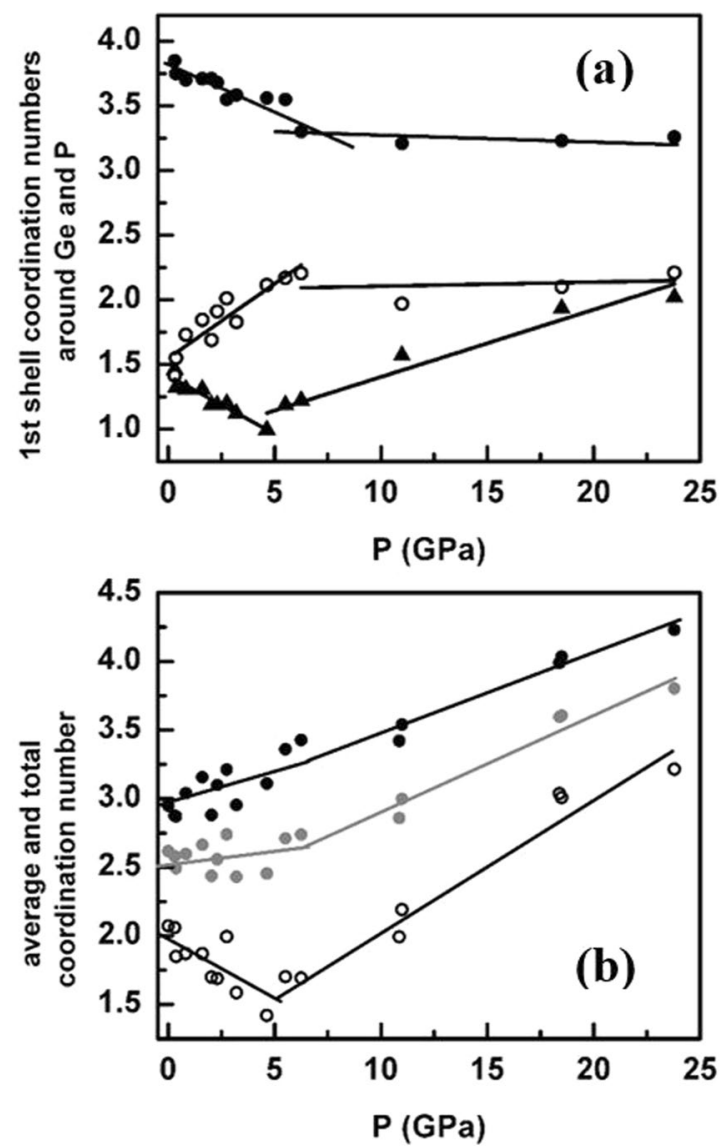

Figure 3. Coordination environments of Ge, P, and Se atoms. (a) Pressure dependence of the $1^{\text {st }}$ shell coordination numbers, $\mathrm{n}_{\mathrm{GeSe}}$ (black dots), $\mathrm{n}_{\mathrm{PP}}$ (open circles), and $\mathrm{n}_{\mathrm{PSe}}$ (triangles) obtained from EPSR simulations. (b) The average (gray dots) and the $1^{\text {st }}$ shell total coordination numbers around $\mathrm{P}$ (black dots) and Se atoms (open circles) as a function of pressure. The lines and curves through the data points in (a) and (b) are guides to the eye only.

The pressure dependence of the nearest-neighbor coordination numbers $\mathrm{n}_{\mathrm{GeSe}}, \mathrm{n}_{\mathrm{PP}}$, and $\mathrm{n}_{\mathrm{PS} \text { e }}$, the total coordination numbers around $\mathrm{P}$ and $\mathrm{Se}$, and the average first shell coordination numbers calculated from the EPSR simulation are shown in Fig. 3a,b. According to the 8-N rule, the coordination numbers of Ge, Se and P atoms are expected to be 4,2 and 3, respectively, which would lead to an average first shell coordination number, $n_{\text {average }}$ of 2.63 for the GPS composition. The results of the EPSR-simulated structure indicate that $\mathrm{n}_{\text {average }}$ for the GPS glass at ambient pressure is $\sim 2.64$. The average value of $\mathrm{n}_{\mathrm{GeSe}}$ is $\sim 3.7$, which however, is somewhat lower than 4.0 for tetrahedral coordination. The total coordination number around the $\mathrm{P}$ atoms is calculated using first shell $\mathrm{P}-\mathrm{P}$ and $\mathrm{P}$-Se correlations and found to be $\sim 3.10$, while that for the Se atoms, calculated using first shell Se-Ge and Se-P correlations yields a value of $\sim 2.10$. The results indicate that $\mathrm{n}_{\text {average }}$ does not change significantly up to $\sim 5.5 \mathrm{GPa}$. Further increase in pressure results in a rapid increase in $\mathrm{n}_{\text {average }}$ to 3.8 at $23.8 \mathrm{GPa}$ (Fig. $3 \mathrm{~b}$ ). This corresponds to a $\sim 46 \%$ increase of $n_{\text {average }}$ in the pressure range of 5.5 to $23.8 \mathrm{GPa}$. The average value of $\mathrm{n}_{\mathrm{GeSe}}$ shows an unusual behavior where it decreases from 3.7 to 3.3 between ambient and $6.3 \mathrm{GPa}$ (Fig. 3a) and then remains practically constant above $6.3 \mathrm{GPa}$. A similar behavior is observed for $\mathrm{n}_{\mathrm{PSe}}$, which decreases from $\sim 1.5$ typical of $\mathrm{P}_{4} \mathrm{Se}_{3}$ molecules at ambient pressure to $\sim 1.0$ at $6.3 \mathrm{GPa}$ and then the trend abruptly reverses with progressive increase above $6.3 \mathrm{GPa}$ and $\mathrm{n}_{\mathrm{PSe}}$ reaches $\sim 2.0$ at $23.8 \mathrm{GPa}$ (Fig. $3 \mathrm{a}$ ). On the other hand, $\mathrm{n}_{\mathrm{PP}}$ increases from $\sim 1.4$ at ambient pressure, as expected for $\mathrm{P}_{4} \mathrm{Se}_{3}$ molecules, to $\sim 2.2$ at $6.3 \mathrm{GPa}$ such that the total $\mathrm{P}$ coordination number at remains $\sim 3.0$ at pressures below $6.3 \mathrm{GPa}$. Further increase in pressure results in almost a nearly invariant $\mathrm{n}_{\mathrm{PP}}$ up to $23.8 \mathrm{GPa}$ such that the total $\mathrm{P}$ coordination number becomes $\sim 4.0$ at the highest pressures (Fig. $3 \mathrm{~b}$ ). The lowering of $\mathrm{n}_{\mathrm{PSe}}$ and $\mathrm{n}_{\mathrm{GeSe}}$ with pressure between ambient and $6.3 \mathrm{GPa}$ is reflected in a drop in the total coordination number for Se from $\sim 2.1$ at ambient to $\sim 1.5$ near $6.3 \mathrm{GPa}$. At higher pressures the densification ultimately results in the formation of $\sim 3$ - and $\sim 4$ - fold coordinated Se and $\mathrm{P}$ atoms, respectively, at $23.8 \mathrm{GPa}$.

The pressure dependence of these various intra- and inter- molecular bond angles is shown in Fig. 4. Again, it is clear from Fig. 4 that the evolution of these bond angles is distinctly different in the low-pressure regime $(<6.3 \mathrm{GPa})$ compared to that in the high-pressure regime $(>6.3 \mathrm{GPa})$. As the pressure increases the Se-P-Se BAD (arises from $\mathrm{P}_{4} \mathrm{Se}_{3}$ molecules where the apical $\mathrm{P}$ atom is linked to three $\mathrm{Se}$ atoms and formed $\mathrm{PSe}_{3}$ pyramids) and $\mathrm{Se}-\mathrm{Ge}-\mathrm{Se} \mathrm{BAD}\left(\mathrm{GeSe}_{4}\right.$ tetrahedral angle) broaden and shift to smaller angles. The rate of this shift increases abruptly at pressures $>6.3 \mathrm{GPa}$. The intra-molecular P-Se-P BAD peak centered at $\sim 100^{\circ}$ (arises from the linkage 

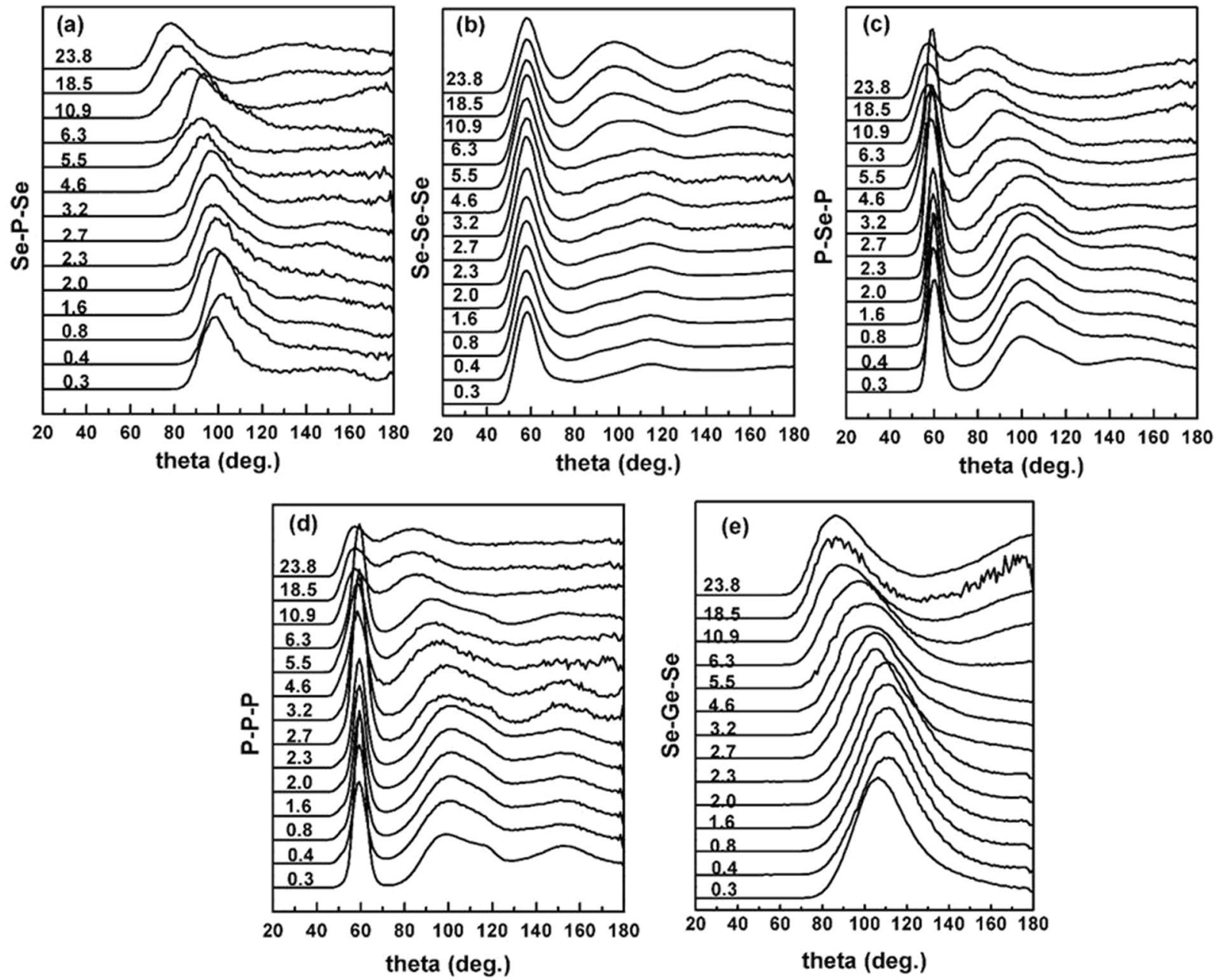

Figure 4. Pressure dependence of bond angle distributions. Pressure dependence of $\mathrm{P}-\mathrm{P}-\mathrm{P}\left(\mathrm{1}^{\text {st }}\right.$ shell $)$, $\mathrm{P}-\mathrm{Se}-\mathrm{P}\left(1^{\text {st }}\right.$ shell $)$, Se-P-Se ( $1^{\text {st }}$ shell $)$, Se-Ge-Se ( $1^{\text {st }}$ shell $)$ and Se-Se-Se $\left(2^{\text {nd }}\right.$ shell $)$ bond angle distributions obtained from the EPSR simulations. Data are vertically offset for clarity and corresponding pressures in GPa are given alongside.

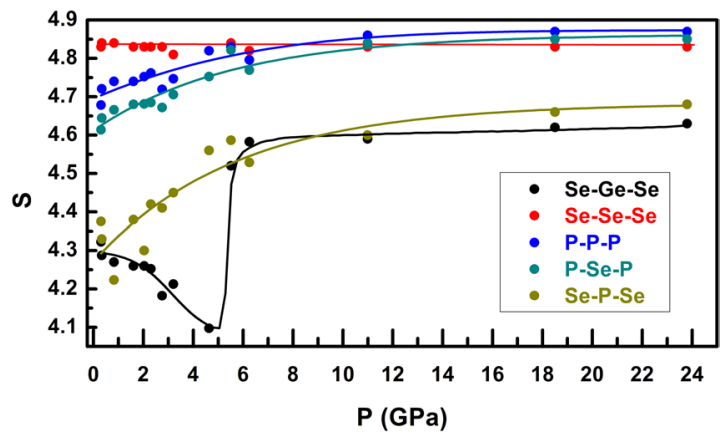

Figure 5. Entropy Information entropy of the bond angle distributions.

of apical $\mathrm{P}$ atom to basal $\mathrm{P}_{3}$ plane via $\mathrm{P}$-Se bonds) displays a similar behavior. The broad peak in the P-P-P distribution (centered in the range of $\sim 80-130^{\circ}$ ) corresponding to the amorphous red-P type structural moieties also displays a rapid shift to lower angles with pressure above $6.3 \mathrm{GPa}$.

The information entropy of the bond angle distribution functions is calculated using $\boldsymbol{S}=-\sum_{\boldsymbol{i}} \boldsymbol{P}_{\boldsymbol{i}} \boldsymbol{l n} \boldsymbol{P}_{\boldsymbol{i}}$, where $P_{i}$ is the distribution of the bond angles. The entropy of the bond angle distribution function is a measure of how well defined the bond angles are at each pressure (large entropy, broad distribution). The pressure dependence of the entropy is depicted in Fig. 5. A very dramatic change in the entropy up to $6.3 \mathrm{GPa}$ is observed for $\mathrm{Se}-\mathrm{Ge}-\mathrm{Se}$ 

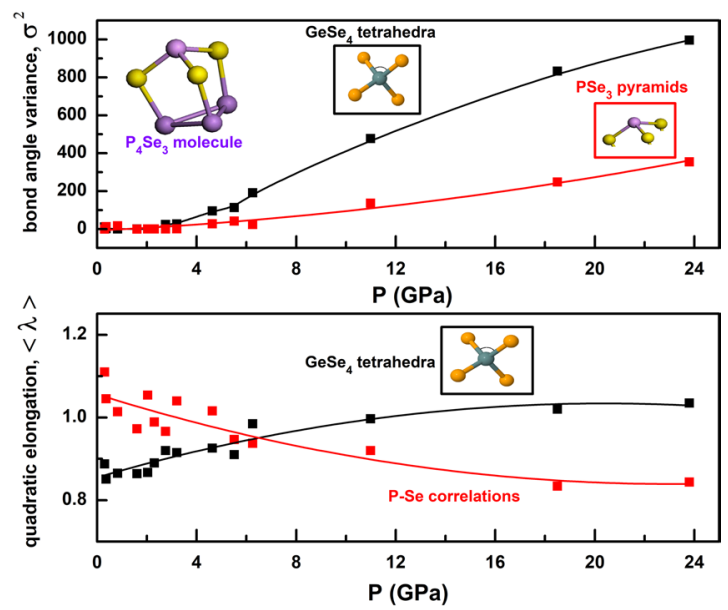

Figure 6. Pressure variation of distortion parameters. Top: Variation of bond-angle variance with pressure for $\mathrm{GeSe}_{4}$ tetrahedral units and $\mathrm{PSe}_{3}$ pyramids in $\mathrm{P}_{4} \mathrm{Se}_{3}$ molecules. Bottom: Pressure dependence of the quadratic elongation of $\mathrm{GeSe}_{4}$ and intra- and inter- molecular P-Se correlations.

and Se-P-Se bond angle distributions, rather than the $\mathrm{P}-\mathrm{P}-\mathrm{P}, \mathrm{P}-\mathrm{Se}-\mathrm{P}$ distributions, implying a fast structural change in the atomic configuration. For each distribution, above $6.3 \mathrm{GPa}$, the curve shows a change in slope, and the growth in $\mathrm{S}$ is slower. Se-Se-Se distribution calculated for second shell neighborhood distances does not alter the information entropy, which implies the stability of Se distribution in the second shell neighborhood. However, it must be noted that only a part of the configurational entropy is associated with that of the bond angle distributions. Therefore, a direct one-to-one correlation between the pressure dependence of the bond angle entropy and that of the degree of molecular-to-network transformation may not be obvious. Nevertheless, the abruptness in the change in the Se-Ge-Se bond angle distribution entropy is the most distinct among the order parameters and indicates a fundamental shift in the densification mechanism. This result is indeed interesting since the presence of $\mathrm{GeSe}_{4}$ clusters is crucial to the glass-forming ability of this molecular liquid.

Distortion of $\mathrm{GeSe}_{4}$ tetrahedral units and $\mathrm{PSe}_{3}$ pyramids in $\mathrm{P}_{4} \mathrm{Se}_{3}$ molecules. The degree of distortion for all molecules is quantified in terms of quadratic elongation (QE) and angle variance (AV). The former gives a measure of the distortion in bond lengths which would all be equal in an ideal polyhedron whereas the latter gives a measure of the distortion of bond angles from their ideal values. The ideal values of QE and AV are unity and zero, respectively. The results are depicted in Fig. 6. QE values have been calculated using the average $\mathrm{Ge}$-Se and P-Se bond-lengths where the former has contribution from $\mathrm{GeSe}_{4}$ tetrahedral units only but the latter gives the distortion in both $\mathrm{P}_{4} \mathrm{Se}_{3}$ intra- and inter- molecular bond lengths. QE results (Fig. 6) suggest the instability of the $\mathrm{GeSe}_{4}$ tetrahedral units. As pressure rises, the degree of distortion slightly increases for $\mathrm{GeSe}_{4}$ tetrahedral units but QE values for $\mathrm{P}_{4} \mathrm{Se}_{3}$ intra- and inter- molecular bonding structure decreases. This can be related to the fact that the P-Se correlations include more complex structure, covering both inter-molecular and intra-molecular P-Se bonds. AV results (Fig. 6), which have been calculated using the Se-Ge-Se (inter-GeSe ${ }_{4}$ tetrahedral) and $\mathrm{Se}-\mathrm{P}-\mathrm{Se}$ ( $\mathrm{PSe}_{3}$ pyramids in $\mathrm{P}_{4} \mathrm{Se}_{3}$ molecules) bond angle distributions, give us a better understanding about the degree of distortion. The $\mathrm{GeSe}_{4}$ tetrahedral shows a much larger distortion level (above $6 \mathrm{GPa}$ ) than $\mathrm{PSe}_{3}$ pyramids do. $\mathrm{GeSe}_{4}$ units remain the most distorted over the pressure range studied.

\section{Discussion}

The $\mathrm{S}(\mathrm{Q})$ and $\mathrm{g}(\mathrm{r})$ at ambient pressure in combination with the EPSR simulation show interatomic distances, coordination numbers, various bond angles and PDFs that are all consistent with a structural scenario that is in excellent agreement with previous studies on this and similar glasses based on ${ }^{31} \mathrm{P}$ NMR and Raman spectroscopy. In this scenario, the GPS glass structure at ambient pressure consists predominantly of $\mathrm{P}_{4} \mathrm{Se}_{3}$ molecules and amorphous red-phosphorus type moieties along with a small concentration of $\mathrm{GeSe}_{4}$ tetrahedra ${ }^{15}$. The bonding of the constituent elements appears to largely obey the $8-\mathrm{N}$ rule. The relative concentrations of these structural units can be estimated by considering that the GPS glass composition can be written as: $\left(\mathrm{GeSe}_{2}\right)_{2.8}+\left(\mathrm{P}_{4} \mathrm{Se}_{3}\right)_{11.3}$ $+(\mathrm{P})_{12.5}$. The lack of any Ge-P nearest-neighbor and Ge-P-Se angular correlations (Supplementary Fig. S2) is indicative of the absence of any significant cross-linking between molecules via Ge atoms. Rather, the presence of a broad but distinct $\mathrm{Ge}-\mathrm{Se}-\mathrm{Ge}$ correlation suggests local clustering of corner-shared $\mathrm{GeSe}_{4}$ tetrahedra in the glass structure. This clustering of Ge atoms is also evident in Supplementary Fig. S4a, showing the spatial distribution of $\mathrm{Ge}$ atoms in a part of the simulation cell. On the other hand, local structural motifs taken from the simulation cell show evidence for amorphous red-phosphorus type moieties enabling cross-linking between a small fraction of $\mathrm{P}_{4} \mathrm{Se}_{3}$ molecules via an opening of their basal $\mathrm{P}_{3}$ ring (Supplementary Fig. S4b).

When taken together, the pressure dependent structural evolution of the $S(Q), G(r)$ and all structural parameters obtained from EPSR simulation yield a consistent scenario of a (predominantly) molecular-to-network polyamorphic structural transformation of the GPS glass across $\sim 6.3 \mathrm{GPa}$. In the low-pressure regime between ambient and $6.3 \mathrm{GPa}$, the structure remains predominantly molecular and densifies via increased molecular 


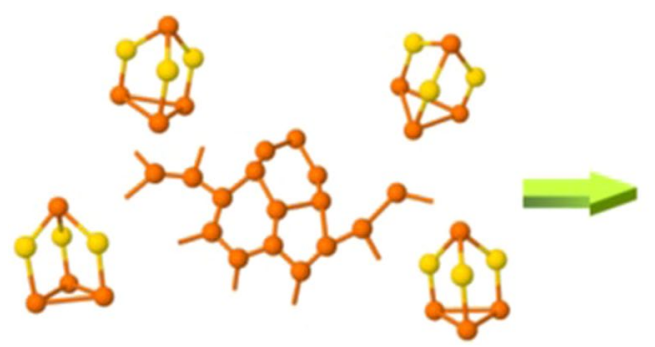

Low-Pressure

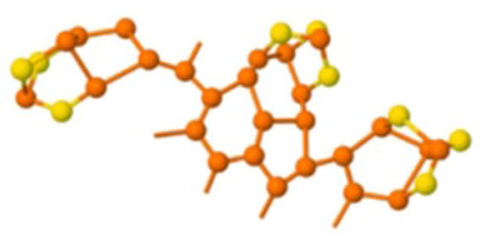

High-Pressure

Figure 7. Cage-opening mechanism in GPS glass. The cage opening of $\mathrm{P}_{4} \mathrm{Se}_{3}$ molecules as a precursor for the network formation. The polyamorphic transformation between low pressure and high pressure structures involves opening of the $\mathrm{P}_{3}$ ring at the base of the $\mathrm{P}_{4} \mathrm{Se}_{3}$ molecules and subsequent reaction with planar edgeshared phosphorus rings.

packing which is initially relatively rapid up to $\sim 2-3 \mathrm{GPa}$ and subsequently slows down at higher pressures up to $~$ $6.3 \mathrm{GPa}$, presumably due to increased intermolecular repulsion. This change in molecular packing rate is evident in the pressure dependence of the positions of the FSDP and the PP (Fig. 1b,c). Increased molecular packing also results in a loss of the intermediate-range ordering associated with inter-molecular correlations which is manifested in the rapid lowering of the intensity of FSDP in this pressure regime. It is rather intriguing to note that, besides increased molecular packing, the P-P coordination number increases from $\sim 1.5$ to $\sim 2.0$ in this pressure regime (Fig. 3a). This result indicates the onset of cage opening for a significant fraction of the $\mathrm{P}_{4} \mathrm{Se}_{3}$ molecules via breaking of the $\mathrm{P}$-Se-P intramolecular linkages between the apical $\mathrm{PSe}_{3}$ pyramid and the basal $\mathrm{P}_{3}$ triangle. This observation is consistent with the results reported in previous in situ high-pressure Raman spectroscopic studies of the $\mathrm{Ge}_{2.5} \mathrm{As}_{51.25} \mathrm{~S}_{46.25}$ glass with $\mathrm{As}_{4} \mathrm{~S}_{3}$ cage molecules that demonstrated complete disappearance of the breathing mode of the basal $\mathrm{As}_{3}$ triangle near $\sim 3 \mathrm{GPa}^{7}$. Once a critical fraction of the molecules undergoes cage opening, the structure collapses into a network near $\sim 6.3 \mathrm{GPa}$ with the simultaneous loss of the FSDP and an abrupt shift in the PP position (Fig. 1c). The densification of this network in the high-pressure regime is marked by rather abrupt changes in the $\mathrm{g}(\mathrm{r})$ and the PDFs as well as in the rate of change of various bond lengths and angles (Figs. 2, 4). Network structures typically densify at high pressures via increase in the coordination numbers of the constituent atoms. This phenomenon is evident in the behavior of the coordination numbers of $\mathrm{P}$ and Se atoms in the GPS glass, which increase in the high-pressure regime to 4 and 3 (from 3 and 2), respectively, at $23.8 \mathrm{GPa}$ (Fig. 3b).

As noted earlier, a pressure-induced molecule-to-network polyamorphic transformation was also reported in the literature for the $\mathrm{Ge}_{2.5} \mathrm{As}_{51.25} \mathrm{~S}_{46.25}$ glass with $\mathrm{As}_{4} \mathrm{~S}_{3}$ cage molecules ${ }^{5,7}$. However, these studies did not directly address the structural mechanism for this transformation. The results obtained in the present study clearly indicate the cage opening of $\mathrm{P}_{4} \mathrm{Se}_{3}$ molecules as a precursor for the network formation. This cage-opening mechanism is schematically shown in Fig. 7. Furthermore, to the best of our knowledge, this is the first report of clear observation of two distinct pressure regimes for the densification of the molecular and network structures which puts polyamorphism in these molecular systems on a firm footing. It is clear that the low pressure molecular structure is more ordered compared to high pressure network structure counterpart, which indicates positive entropy and negative volume changes associated with the transformation. Consequently, the slope of the P-T phase boundary should be negative. This hypothesis would imply that the transition could be observed at ambient pressure upon increasing temperature. Future measurements of the viscosity of the $\mathrm{Ge}_{2.5} \mathrm{As}_{51.25} \mathrm{~S}_{46.25}$ liquid over a wide temperature range may reveal the presence of such transformations in the equilibrium liquid state. It is to be noted that recent studies indeed suggest a liquid-liquid phase transition at ambient pressure with increasing temperature can be observed in the form of a sudden change in the temperature dependence of viscosity ${ }^{16-18}$. In fact, the recent proposal of possible strong-to-fragile transitions in glass-forming liquids with increasing temperature ${ }^{18}$ may be connected to their pressure-dependent counterparts in polyamorphic transitions with negative P-T slopes.

\section{Methods}

Sample preparation. The GPS glass was synthesized by melting a mixture of the starting elements $\left(\geq 99.9995 \%\right.$ purity in metals basis) in an evacuated ( $10^{-6}$ Torr) fused silica ampoule at $873 \mathrm{~K}$ for at least $13 \mathrm{~h}$ in a rocking furnace. The ampoule was rapidly quenched in water to make transparent orange-red glass. The glass sample was confirmed to be amorphous using powder x-ray diffraction. The composition reported above was obtained from electron probe micro-analysis. The small amount of $\mathrm{Ge}$ doping and P-excess (over the $\mathrm{P}_{4} \mathrm{Se}_{3}$ stoichiometry) are necessary in stabilizing this glass against devitrification. The density $\left(3.09 \mathrm{gcm}^{-3}\right)$, glass transition temperature $\mathrm{T}_{\mathrm{g}}(468 \mathrm{~K})$, Raman and ${ }^{31} \mathrm{P}$ NMR spectra of this glass were reported in a previous publication ${ }^{8}$.

X-ray diffraction and absorption. High pressure/room temperature (hprt-XRD) $\mathrm{x}$-ray diffraction data were collected on beamline 12.2.2 at the Advanced Light Source (ALS) ${ }^{19}$. An x-ray energy of $30 \mathrm{keV}(\lambda$ $=0.4133 \AA$ ) was selected to collect the hprt-XRD data. A single $\mathrm{x}$-ray wavelength was selected using a $\operatorname{Si}(111)$ double crystal monochromator (12.2.2, ALS). The $\mathrm{x}$-ray beam was focused to a $10 \times 10 \mu \mathrm{m}$ spot size at the sample position. X-ray diffraction images were collected using a MAR345 image plate detector. The sample detector distance and the detector tilt angles were measured using powder diffraction from a $\mathrm{LaB}_{6}$ standard. The $\mathrm{x}$-ray beam was $99 \%$ horizontally polarized and all geometric and polarization corrections were made during the angular integration using the FIT2 $\mathrm{D}^{20}$ software package. Details of the $\mathrm{x}$-ray diffraction data reduction process 
to obtain the structure factors, $S(\mathrm{Q})$ were described in our previous study ${ }^{21}$. A typical process was exemplified in Supplementary Fig. S5 for the X-ray diffraction data collected at $0.4 \mathrm{GPa}$. For absorption measurements and determination of densities we followed the method described by Shen et al. ${ }^{22}$. A rhenium gasket pre-indented to thickness of $\sim 22 \mu \mathrm{m}$ was used between diamond anvils. Two chambers with $\sim 90 \mu \mathrm{m}$ diameter were formed by laser-drilling, and serve as cavities for sample material (loaded into one of the holes) and $\mathrm{NaCl}$ (loaded into the other hole). Absorption measurements were performed in the pressure range of $0-25 \mathrm{GPa}$ using a $10 \mu \mathrm{m} \mathrm{x}$-ray beam with the energy of $20 \mathrm{keV}(\lambda=0.6199 \AA)$. The $\mathrm{x}$-ray intensity transmitted through each hole was measured using a pin-diode with the uncertainty of \pm 0.05 (see Supplementary Fig. S6). By measuring the transmitted intensities, gasket thickness and sample density have been obtained as $22.1 \pm 1.7 \mu \mathrm{m}$ and $3.09 \pm 0.18 \mathrm{~g} / \mathrm{cm}^{3}$, and $10.2 \pm 0.2 \mu \mathrm{m}$ and $4.66 \pm 0.60 \mathrm{~g} / \mathrm{cm}^{3}$ (see Supplementary Fig. S7 for the full list in 0-25 GPa pressure range) at $0.3 \mathrm{GPa}$ and $25 \mathrm{GPa}$, respectively. The uncertainties were determined by the errors in the $\mathrm{x}$-ray transmission intensities and in the unit cell volumes obtained by diffraction. The standard errors reported here are $( \pm 0.60 \mathrm{~g} /$ $\mathrm{cm}^{3}$ maximum) relatively bigger than the ones obtained in ref. ${ }^{22} .\left( \pm 0.11 \mathrm{~g} / \mathrm{cm}^{3}\right)$, which indicates considerable irregularities in transmission profile (Supplementary Fig. S6).

High pressure generation. High pressures were generated using a symmetric diamond anvil cell (DAC) equipped with $400 \mu \mathrm{m}$ culet diamonds, c-BN seats and a rhenium gasket pre-indented to a thickness of $27 \mu \mathrm{m}$. A $100 \mu \mathrm{m}$ hole was drilled in the center of the indentation, and then loaded with sample, two spheres of ruby as a pressure marker, and a 4:1 methanol: ethanol mixture as the pressure-transmitting fluid. The ruby fluorescence spectra showed a well resolved doublet for all measurements we performed, which leads us to conclude that the sample is compressed in fairly hydrostatic pressure environment.

3D structural modeling. Empirical Potential Structure Refinement (EPSR) method ${ }^{23}$ was used to determine the atomic structure of GPS glass at ambient and high pressure. For each simulation, $56 \mathrm{Ge}, 1154 \mathrm{P}$ and $790 \mathrm{Se}$ atoms were mixed in a cubic simulation box with a volume constrained by the experimentally determined glass density (Supplementary Tables S1, S2). The Lennard-Jones potential well depth parameters and range parameters were set to $0.1 \mathrm{~kJ} / \mathrm{mol}$ and $2.0 \AA, 0.5 \mathrm{~kJ} / \mathrm{mol}$ and $2.2 \AA, 0.1 \mathrm{~kJ} / \mathrm{mol}$ and $2.0 \AA$ for Ge, $\mathrm{P}$ and Se, respectively ${ }^{21,24}$. Minimum approach distances listed in (Supplementary Table S1) were used to constrain the modelling of experimental S(Q).

Received: 12 November 2019; Accepted: 3 March 2020;

Published online: 23 March 2020

\section{References}

1. McMillan, P. F. et al. Polyamorphism and liquid-liquid phase transitions: challenges for experiment and theory. J. Physics: Condensed Matter 19, 415101 (2007)

2. Mishima, O. Polyamorphism in water. Proc. Jpn. Acad. Ser. B. Phys. Biol. Sci. 86(3), 165-175 (2010).

3. Klotz, S. et al. Nature of the polyamorphic transition in ice under pressure. Phys. Rev. Lett. 94, 025506 (2005).

4. Debenedetti, P. G. \& Stanley, H. E. Supercooled and glassy water. Physics Today 56(6), 40 (2003).

5. Sen, S., Gaudio, S., Aitken, B. G. \& Lesher, C. E. Observation of a pressure-induced first-order polyamorphic transition in a chalcogenide glass at ambient temperature. Phys. Rev. Lett. 97, 025504 (2006).

6. Soyer-Uzun, S. et al. In situ high-pressure x-ray diffraction study of densification of a molecular chalcogenide glass. J. Phys. and Chem. of Solids 69, 2336-2340 (2008).

7. Kalkan, B. et al. Hysteretically reversible phase transition in a molecular glass. J. Chem. Phys. 137, 224503 (2012).

8. Gjersing, E. L., Sen, S. \& Aitken, B. G. Molecular dynamics in supercooled P-Se liquids near the glass transition: results from ${ }^{31} \mathrm{P}$ NMR spectroscopy. J. Phys. Chem. B 115, 2857-2863 (2011).

9. Verrall, D. J. \& Elliot, S. R. Experimental evidence for an inorganic molecular glass. Phys. Rev. Lett. 61, 974-977 (1988).

10. Bytchkov, A., Fayon, F., Massiot, D., Hennet, L. \& Price, D. L. ${ }^{31}$ P solid-state NMR studies of the short-range order in phosphorusselenium glasses. Phys. Chem. Chem. Phys. 12, 1535-1542 (2010).

11. Soyer-Uzun, S., Sen, S. \& Aitken, B. G. Network vs molecular structural characteristics of Ge-doped arsenic sulfide glasses: a combined neutron/x-ray diffraction, extended $\mathrm{x}$-ray absorption fine structure, and raman spectroscopic study. J. Phys. Chem. C 113, 6231-6242 (2009).

12. Wright, A. C. et al. Neutron studies of an inorganic plastic glass. J. Non-Cryst. Solids 357, 2502-2510 (2011).

13. Salmon, P. S., Martin, R. A., Mason, P. E. \& Cuello, G. J. Topological versus chemical ordering in network glasses at intermediate and external length scales. Nature 435, 75-78 (2005).

14. Bytchkov, A. et al. Intermediate- and short-range order in phosphorus-selenium glasses. Phys. Rev. B 83, 144201 (2011)

15. Kalkan, B., Benmore, C. J., Aitken, B. G., Sen, S. \& Clark, S. M. A comparative study of the atomic structures of Ge-doped As4S3 and P4Se3 molecular glasses. J. Non-Cryst. Solids 514, 83-89 (2019).

16. Brazhkin, V. V. et al. AsS melt under pressure: One substance, three liquids. Phys. Rev. Lett. 100, 145701 (2008).

17. Brazhkin, V. V., Kanzaki, M., Funakoshi, K. \& Katayama, Y. Viscosity behavior spanning four orders of magnitude in As-S melts under high pressure. Phys. Rev. Lett. 102, 115901 (2009).

18. Zhang, C., Hu, L., Yue, Y. \& Mauro, J. C. Fragile-to-strong transition in metallic glass-forming liquids. J. Chem. Phys. 133, 014508 (2010).

19. Kunz, M. et al. A beamline for high-pressure studies at the Advanced Light Source with a superconducting bending magnet as the source. J. Synchrotron Radiat. 12, 650 (2005).

20. Hammersley, A. P., Svensson, S. O., Hanfland, M., Fitch, A. N. \& Hausermann, D. Two-dimensional detector software: from real detector to idealized image or two-theta scan. High Pressure Research 14, 235 (1996).

21. Kalkan, B., Dias, R. P., Yoo, C.-S., Clark, S. M. \& Sen, S. Polyamorphism and pressure-induced metallization at the rigidity percolation threshold in densified $\mathrm{GeSe}_{4}$ glass. J. Phys. Chem. C 118, 10 (2014).

22. Shen, G. et al. Melting Studies of Indium: Determination of Structure and Density of Melts at High Pressures and High Temperatures. J. Phys. Cond. Matt. 14, 10533-10540 (2002).

23. Soper, A. K. Partial structure factors from disordered materials diffraction data: An approach using empirical potential structure refinement. Phys. Rev. B 72, 104204/1-104204/12 (2005).

24. Zaug, J. M., Soper, A. K. \& Clark, S. M. Pressure-dependent structures of amorphous red phosphorus and the origin of the first sharp diffraction peaks. Nature Mat. 7, 890-899 (2008) 


\title{
Acknowledgements
}

The Advanced Light Source is supported by the Director, Office of Science, Office of Basic Energy Sciences, of the U.S. Department of Energy under Contract No. DE-AC02-05CH11231, and by COMPRES, the Consortium for Materials Properties Research in Earth Sciences under NSF Cooperative Agreement EAR 10-43050. B.K. thanks J. Knight for support during the ALS beamtime. S.M.C thanks J. M. Zaug for loan of the ultrawide-aperture DAC used for the x-ray scattering data collection. The work at UCD was supported by an NSF grant DMR-1855176.

\section{Author contributions}

S.S., S.M.C. and B.K. designed the research; B.G.A. synthesized the samples; B.K. and S.M.C. performed the synchrotron x-ray diffraction and absorption experiments; B.K. and G.O. reduced the X-ray scattering data; B.K. performed EPSR modelling. All authors reviewed the results and wrote the paper.

\section{Competing interests}

The authors declare no competing interests.

\section{Additional information}

Supplementary information is available for this paper at https://doi.org/10.1038/s41598-020-61997-x.

Correspondence and requests for materials should be addressed to B.K.

Reprints and permissions information is available at www.nature.com/reprints.

Publisher's note Springer Nature remains neutral with regard to jurisdictional claims in published maps and institutional affiliations.

\begin{abstract}
(c) (i) Open Access This article is licensed under a Creative Commons Attribution 4.0 International License, which permits use, sharing, adaptation, distribution and reproduction in any medium or format, as long as you give appropriate credit to the original author(s) and the source, provide a link to the Creative Commons license, and indicate if changes were made. The images or other third party material in this article are included in the article's Creative Commons license, unless indicated otherwise in a credit line to the material. If material is not included in the article's Creative Commons license and your intended use is not permitted by statutory regulation or exceeds the permitted use, you will need to obtain permission directly from the copyright holder. To view a copy of this license, visit http://creativecommons.org/licenses/by/4.0/.
\end{abstract}

(C) The Author(s) 2020 